\title{
Is there a benefit for a care protocol in the treatment of septic shock?
}

\author{
Ilaria Emanuela Bossi • Anna Maria Rusconi • \\ Geoff Lampard • Michael Szava-Kovats • \\ Gruppo di Autoformazione Metodologica (GrAM)
}

Received: 31 October 2014/Accepted: 5 November 2014/Published online: 27 November 2014

(C) SIMI 2014

\section{Background}

Severe sepsis and septic shock have a short-term mortality in excess of $20 \%$ [1]. In 2001, a single-center ED-based study of a 6-hour early goal-directed therapy (EGDT) protocol reported a significant reduction in in-hospital mortality (30.5 vs $46.5 \%$ ) [2]. This EGDT protocol mandated early central venous catheterization to monitor central venous pressure, central venous oxygen saturation, and to guide use of intravenous fluids, vasopressors and packed red cell transfusions.

Many trials have been published in the subsequent decade raising the question of whether all elements of the protocol are necessary.

\section{Summary}

The ProCESS (Protocolized Care for Early Septic Shock) trial was conducted to assess whether a protocol-based resuscitation for septic shock treatment was superior to usual care, and to evaluate the need for a protocol that includes central hemodynamic monitoring to guide the use of fluids, vasopressors, blood transfusions and dobutamine [3].

ProCESS is a randomized trial conducted in 31 tertiarycare hospitals in the United States of America.

\footnotetext{
I. E. Bossi $(\bowtie) \cdot$ A. M. Rusconi

Emergency Medicine, San Anna Hospital, Como, Italy

e-mail: ilariaiaia@yahoo.com

G. Lampard · M. Szava-Kovats

Emergency Medicine, University of Calgary, Calgary, Canada
}

Study inclusion criteria were: over 18 years of age, sepsis suspected by treating physician, and presence of septic shock, as defined by two or more systemic inflammatory response syndrome (SIRS) criteria, [4] with either hypotension refractory to intravenous fluid resuscitation, or a serum lactate greater than $4 \mathrm{mmol} / \mathrm{L}$.

Patients did not have to be in shock on arrival in the emergency department (ED), but had to be enrolled in the study in ED within $2 \mathrm{~h}$ after the earliest detection of shock, and within $12 \mathrm{~h}$ after arrival.

Patients were randomly assigned to one of the three groups for $6 \mathrm{~h}$ of resuscitation:

1. Protocol-based EGDT: the resuscitation team followed the previously described "Rivers' protocol," with placement of a central venous catheter to monitor pressure and $\mathrm{Scv02}$, and to administer intravenous fluid, vasopressors, dobutamine, or packed red cell transfusions.

2. Protocol-based "standard therapy:" the resuscitation team adhered to a less invasive novel 6-hour sepsis protocol developed by the ProCESS investigators after a literature review and two independent surveys of intensivist and emergency physician practices worldwide. It required adequate peripheral venous access and administration of fluids and vasoactive agents to reach goals for systolic blood pressure or shock index. The protocol recommended transfusion only if the hemoglobin level was less than $7.5 \mathrm{~g} / \mathrm{dL}$.

3. Usual care: the bedside physician directed all care, with the study coordinator collecting data, but not prompting any actions.

The primary outcome was all-cause in-hospital mortality at 60 days. Secondary outcomes included all-cause mortality at 90 days and 1 year duration of cardiovascular, respiratory, and renal failure requiring invasive supportive 
care, duration of hospitalization and ICU stay, serious adverse events, and hospital discharge disposition. Outcomes were assessed using an intention-to-treat analysis.

From March 2008 through May 2013, 1,341 patients were enrolled: 439 in the protocol-based EGDT group, 446 in the protocol-based "standard therapy," and 456 in the usual care group. There was no significant 60 -day mortality difference, with $21.0,18.2$, and $18.9 \%$ of patients dead at 60 days in the respective groups (protocol-based EGDT vs usual care $\mathrm{RR}=1.04,95 \%$ CI $0.82-1.31 ; p=0.83$ ), (protocol-based EGDT vs protocol-based standard care $\mathrm{RR}=1.15,95 \%$ CI $0.88-1.51 ; p=0.31)$. There was also no significant difference in 90 -day or 1-year mortality.

Resuscitation strategies differed between groups. Intravenous fluid administration from randomization to hour 6 in the EGDT, standard protocol-based therapy, and usual care groups was 2.8 vs 3.3 vs $2.3 \mathrm{~L}$, respectively $(p<0.001)$. Vasopressor use was higher in the protocolbased groups (54.9 vs 52.2 vs $44.1 \%$, respectively; $p=0.003$ ). Patients in the EGDT group received significantly higher rates of dobutamine administration (8.0 vs 1.1 vs $0.9 \%$, respectively; $p<0.001$ ), and packed red cell transfusions (14.4 vs 8.3 vs $7.5 \%$, respectively; $p=0.001$ ), when compared to the other two groups.

The use of antibiotics, glucocorticoids and activated protein $\mathrm{C}$ was similar across the three groups. The use of intravenous fluids, vasopressors, dobutamine and blood transfusion between 6 and $72 \mathrm{~h}$ did not differ significantly among the groups.

\section{Strengths of the study}

- It deals with a very relevant clinical problem.

- ProCESS is a large multicenter study involving 31 different EDs, and enrolling 1,341 patients.

\section{Weakness of the study}

- Patients screening identified 3,843 eligible patients, but about two-thirds of these $(2,492)$ were excluded for logistical reasons, reduced mental capacity or refusal to participate in the study. Such a high percentage of patients eligible but excluded, might have reduced the external validity of the study.

\section{Question marks}

- There is a great difference in mortality comparing Rivers trial $(30 \%$ in EGDT vs $46 \%$ in usual care group), with ProCESS (18-21\%). Patients in the original Rivers et al. trial were older, had more chronic conditions, had higher admission lactate levels and were all in septic shock, otherwise in ProCESS, patients could not be in shock on arrival. We question if the study populations might have been different.

- Therapies provided in the Rivers' et al. trial were markedly different than the ProCESS EGDT group. There were lower rates of blood transfusion (14 vs $64 \%$ ), higher rates of vasopressor use (55 vs $27 \%$ ), and lower rates of mechanical ventilation (26 vs $53 \%$ ). We wonder if these differences can be due to a different application of the treatment protocol, or to a difference in the kind of enrolled patients.

- Patients in the Rivers' trial were identified upon arrival to the ED. In ProCESS, patients could be identified up to $12 \mathrm{~h}$ post-arrival. It would be interesting to know how many patients enrolled in the ProCESS trial were in septic shock on arrival in the ED, and how many patients later met the inclusion criteria. Enrollment of patients who subsequently met criteria after arrival might have altered the severity of sepsis in the ProCESS patients compared to the patients in the Rivers et al. trial.

- The compliance with the protocol in the EGDT group was quite high but not perfect (incomplete compliance in $11.9 \%$ of patients at $6 \mathrm{~h}$ ). In the EGDT group, central venous catheterization occurred in only $60 \%$ of the patients at $1 \mathrm{~h}$, in $85 \%$ at $2 \mathrm{~h}$ and in $93.6 \%$ at $6 \mathrm{~h}$. Moreover, central venous catheterization occurred in 56.5 and $57.9 \%$ of the patients at $6 \mathrm{~h}$ in the other two treatment groups. At last, to be eligible, the study sites had to comply with the Surviving Sepsis Campaign guidelines [5] for non-resuscitation aspects of care. We wonder if these factors might have diluted the treatment differences in the three groups.

- $76 \%$ of patients received antibiotics, and on average, $2 \mathrm{~L}$ of fluid prior to randomization. This is in keeping with current Surviving Sepsis Campaign recommendations [5]. We question if a benefit for a care protocol would be more likely in non-tertiary level care centers where standard care may be less aggressive.

- It would be interesting to have more information about timing of the administration and appropriateness of antibiotic therapy performed, since we know that this treatment significantly affects prognosis in septic patients.

- Time has passed since the River's publication. We question if in more than 10 years the usual care in the management of septic shock could have been improved compared with usual care at the time of the Rivers' study. Moreover, in over 10 years after the Rivers' study and after the Survival Sepsis Campaign, we 
wonder if even the threshold of suspicion and caution with respect to sepsis has improved, and has led to earlier recognition and treatment of septic patients.

\section{Sponsorship}

The ProCESS trial was supported by a grant from the National Institute of General Medical Sciences, National Institutes of Health. Edwards Lifesciences supported the ProCESS trial with the Scv02 monitoring equipment. The company had no other role in the study.

\section{Clinical bottom line}

In patients with septic shock diagnosed in the ED, a protocol for EGDT based on invasive monitoring devices does not appear to confer a survival benefit when compared with current care resuscitation. Given the large discrepancy in mortality between the ProCESS trial and the Rivers' one, further studies on care protocol are warranted.

\section{References}

1. Angus DC, van der Poll T (2013) Severe sepsis and septic shock. N Engl J Med 369(21):2063

2. Rivers E, Nguyen B, Havstad S et al (2001) Early goal-directed therapy in the treatment of severe sepsis and septic shock. N Engl J Med 345(19): 1368-1377

3. Yealy DM, Kellum JA, Huang DT et al (2014) A randomized trial of protocol-based care for early septic shock. N Engl J Med 370(18):1683-1693

4. Bone RC, Balk RA, Cerra FB et al (2009) Definitions for sepsis and organ failure and guidelines for the use of innovative therapies in sepsis. The ACCP/SCCM Consensus Conference Committee. American College of Chest Physicians/Society of Critical Care Medicine. 1992. Chest 101(6):1644-1655

5. Dellinger RP, Levy MM, Rhodes A et al (2013) Surviving Sepsis Campaign: International guidelines for management of severe sepsis and septic shock: 2012. Crit Care Med 41:580-637

Conflict of interest None. 